\title{
The Alzheimer's Disease Neuroimaging Initiative positron emission tomography core
}

\author{
William J. Jagust ${ }^{\mathrm{a}, \mathrm{b}, *}$, Dan Bandy ${ }^{\mathrm{c}, \mathrm{d}, \mathrm{e}}$, Kewei Chen ${ }^{\mathrm{c}, \mathrm{d}, \mathrm{e}, \mathrm{f}}$, Norman L. Foster ${ }^{\mathrm{g}}$, \\ Susan M. Landau ${ }^{\mathrm{a}, \mathrm{b}}$, Chester A. Mathis ${ }^{\mathrm{h}}$, Julie C. Price ${ }^{\mathrm{h}}$, Eric M. Reiman ${ }^{\mathrm{c}, \mathrm{d}, \mathrm{e}}$, \\ Daniel Skovronsky ${ }^{\mathrm{i}, \mathrm{j}}$, Robert A. Koeppe ${ }^{\mathrm{k}}$; and the Alzheimer's Disease Neuroimaging Initiative ${ }^{1}$ \\ ${ }^{a}$ Helen Wills Neuroscience Institute, University of California Berkeley, Berkeley, CA, USA \\ ${ }^{b}$ Lawrence Berkeley National Laboratory, Berkeley, CA, USA \\ 'Banner Alzheimer's Institute and Banner Good Samaritan PET Center, Phoenix, AZ, USA \\ ${ }^{d}$ Translational Genomics Research Institute, University of Arizona, Phoenix, AZ, USA \\ 'Arizona Alzheimer's Consortium, Phoenix, AZ, USA \\ ${ }^{f}$ Department of Mathematics, Arizona State University, Tempe, AZ, USA \\ ${ }^{g}$ Center for Alzheimer's Care, Imaging and Research and Department of Neurology, University of Utah, Salt Lake City, UT, USA \\ ${ }^{h}$ Department of Radiology, University of Pittsburgh, Pittsburgh, PA, USA \\ ${ }^{i}$ Avid Radiopharmaceuticals, Inc, Philadelphia, PA, USA \\ ${ }^{j}$ Department of Radiology, University of Pennsylvania, Philadelphia, PA, USA \\ ${ }^{k}$ Division of Nuclear Medicine, Department of Radiology, University of Michigan, Ann Arbor, MI, USA
}

Abstract

\begin{abstract}
Background: This is a progress report of the Alzheimer's Disease Neuroimaging Initiative (ADNI) positron emission tomography (PET) Core.

Methods: The Core has supervised the acquisition, quality control, and analysis of longitudinal $\left[{ }^{18}\right.$ F]fluorodeoxyglucose PET (FDG-PET) data in approximately half of the ADNI cohort. In an "add on" study, approximately 100 subjects also underwent scanning with $\left[{ }^{11} \mathrm{C}\right]$ Pittsburgh compound B PET for amyloid imaging. The Core developed quality control procedures and standardized image acquisition by developing an imaging protocol that has been widely adopted in academic and pharmaceutical industry studies. Data processing provides users with scans that have identical orientation and resolution characteristics despite acquisition on multiple scanner models. The Core labs have used many different approaches to characterize differences between subject groups (Alzheimer's disease, mild cognitive impairment, controls), to examine longitudinal change over time in glucose metabolism and amyloid deposition, and to assess the use of FDG-PET as a potential outcome measure in clinical trials. Results: ADNI data indicate that FDG-PET increases statistical power over traditional cognitive measures, might aid subject selection, and could substantially reduce the sample size in a clinical trial. Pittsburgh compound B PET data showed expected group differences, and identified subjects with significant annual increases in amyloid load across the subject groups. The next activities of the PET core in ADNI will entail developing standardized protocols for amyloid imaging using the $\left[{ }^{18} \mathrm{~F}\right]$-labeled amyloid imaging agent AV45, which can be delivered to virtually all ADNI sites.

Conclusions: ADNI has demonstrated the feasibility and utility of multicenter PET studies and is helping to clarify the role of biomarkers in the study of aging and dementia.
\end{abstract}

(C) 2010 The Alzheimer's Association. All rights reserved.

GE Healthcare holds a license agreement with the University of Pittsburgh based on the PIB technology described in this manuscript. Dr Mathis is a coinventor of PIB and, as such, has a financial interest in this license agreement. GE Healthcare had no role in the design or interpretation of results or preparation of this manuscript.

${ }^{1}$ Data used in the preparation of this article were obtained in part from the Alzheimer's Disease Neuroimaging Initiative (ADNI) database (www.loni.
ucla.edu/ADNI). As such, the investigators within ADNI contributed to the design and implementation of ADNI and/or provided data but did not participate in analysis of writing of this report. ADNI investigators are listed at www.loni. ucla.edu/ADNI/Collaboration/ADNI_Authorship.list.pdf

*Corresponding author. Tel.: 510-643-6537.

E-mail address: jagust@berkeley.edu 


\section{Introduction}

Alzheimer's Disease Neuroimaging Initiative (ADNI) was initially conceived as a project designed to help speed drug development by establishing imaging as a reliable and valid biomarker for Alzheimer's disease (AD). As such, the major goal of the positron emission tomography (PET) component of ADNI was to show that $\left[{ }^{18} \mathrm{~F}\right]$ fluorodeoxyglucose (FDG)-PET could serve as an outcome measure to track a drug effect that would provide greater statistical power than routine clinical measures. This required standardization of data acquisition and processing to permit the sort of multicenter data collection that would be necessary for clinical trials. These initial goals have largely been met. Procedures for uploading data, quality control and, most importantly, processing of images to produce final scans with identical image characteristics have all been implemented successfully. These data have been analyzed by multiple laboratories, including analyses by ADNI PET Core laboratories that specifically addressed issues of using FDG-PET as a biomarker for clinical trial outcomes. These results will be discussed later in the text.

Soon after the initiation of the project, it became clear that imaging the $A \beta$ protein in fibrillar, aggregated forms using PET was a biomarker of increasing importance. For this reason, an "add on" project was initiated in the second year of ADNI, which utilized the tracer $\left[{ }^{11} \mathrm{C}\right]$ Pittsburgh compound $\mathrm{B}$ (PIB). Because of the short half-life of $\left[{ }^{11} \mathrm{C}\right](20.4$ minutes) this tracer requires a radiochemistry program and cyclotron, thus only 14 ADNI sites could participate, enrolling 103 subjects. Nevertheless, a major benefit of ADNI has been the ability to obtain longitudinal follow-up as well as multiple biomarker measurements in the same subjects, so that the PIB data have already proven useful and interesting and paved the way for new studies of biomarkers. The availability of PET amyloid imaging agents labeled with the longer half-life (109.7 minutes) $\left[{ }^{18} \mathrm{~F}\right]$ will provide a way of bringing amyloid imaging to all ADNI PET sites, and these studies are planned for the ensuing phases of the project.

As ADNI has evolved, interest in the use of biomarkers has expanded from a role as an outcome measure to a role in subject selection. This is driven largely by the interest in enrolling subjects with very mild AD for clinical trials, as many agree that effective therapy will likely require very early treatment. This is a problematic situation, however, because many individuals with mild symptoms will show little or no cognitive or functional decline over the course of a clinical trial. Thus, selection of subjects with "positive" biomarkers (reflecting AD pathology or more advanced disease) may be a way of enriching a clinical trial cohort for those subjects who are more likely to decline over a short period. ADNI has therefore grown into a broad-based study of $\mathrm{AD}$ biomarkers that may be useful for both subject selection and outcome measures. We review some of the major achievements of the ADNI PET core as well as future plans later in the text.

\section{Data acquisition and processing}

ADNI was launched in 2003 by the National Institute on Aging, the National Institute of Biomedical Imaging and Bioengineering, the Food and Drug Administration, and private pharmaceutical companies and nonprofit organizations. ADNI is the result of efforts of many coinvestigators from a broad range of academic institutions and private corporations, and subjects have been recruited from over 50 sites across the United States and Canada. For up to date information, see www.adni-info.org.

The ADNI PET core has pioneered the development of standards for quality control and acquisition of FDG-PET and PIB-PET data that are now widely utilized in both academic and pharmaceutical studies worldwide. The key design features of this protocol include: (1) simplicity, permitting multisite application; (2) compatibility with current commercially marketed PET cameras; (3) flexible data acquisition for postprocessing to standardize formats, intensity and resolution; (4) low radiation dose; (5) high patient acceptance; (6) real-time quality assurance and quality control; and (7) centralized storage and standardization of images acquired on different scanners. The protocol has been successfully implemented at all ADNI PET sites in North America and has been adopted by several pharmaceutical clinical trials and the Japanese ADNI. The protocol was developed collaboratively among ADNI investigators, with input from the sites and an outside panel of experts. In addition, in the PIB "add-on" we developed a standardized protocol for the acquisition of PIB-PET data that has also been widely adopted.

During the first 2 years of the project, sites were approved for PET scanning by performing a pair of phantom scans on the 3-D Hoffman brain phantom following a protocol that matched the acquisition and reconstruction parameters to be used for the human phase of the ADNI project and electronically transmitting these images to the Laboratory of Neuroimaging at the University of California, Los Angeles. Scans were passed through a quality control process that checked for statistical noise, agreement with a digital version of that phantom (the gold standard), and assessed for image resolution and image uniformity. This process also helped develop a method for assessing and correcting for differences in PET images across sites [1]. There are a total of 17 different scanner models from three vendors that have been used by participating ADNI sites. Intrinsic resolution differs by more than a factor of two across scanners, from as high as $3-4 \mathrm{~mm}$ to as low as $8 \mathrm{~mm}$ effective resolution, thus requiring assessment of resolution and partial volume effects

Human PET studies were first acquired at the start of the second year of ADNI, September 2005. All human PET scans are run through a stringent quality control procedure to assess image quality. Quality control checks include the number of detected coincidence events (for statistical quality), motion assessment across temporal frames, checking for full coverage of the brain, visual checks of images to 
look for the most common PET artifacts (such as normalization problems or motion between attenuation and emission scans), as well as image header checks to make sure the exact ADNI protocol has been followed. Half the enrolled ADNI subjects underwent FDG-PET, and we now have 404 baseline scans, 368 6-month scans, 336 12-month scans, 154 18-month scans (mild cognitive impairment [MCI] subjects only), 283 24-month scans, and to-date 106 36-month scans (MCI and control subjects only). Besides FDG, approximately one quarter of the PET subjects $(\mathrm{n}=103)$ also received PIB scans to image amyloid deposition. Repeat scans at a 1year interval have been performed on 80 of the 103 subjects, while 34 subjects have had a third annual PIB scan.

The original "raw" PET image sets are uploaded to the Laboratory of NeuroImaging (LONI) image repository. Routines read and convert PET images sets to a standard Digital Imaging and Communications in Medicine file format. The different temporal frames are coregistered, and both a dynamic image set and a single-frame averaged image set are produced in the original patient orientation and in the original intrinsic in-plane pixel size and plane spacing for that scanner. In addition, all images sets are reoriented to a common spatial orientation and interpolated onto a uniform image grid ( $1.5 \mathrm{~mm}^{3}$ voxels). In the final step, images are smoothed with a scanner-specific filter derived from each site's Hoffman phantom [1] to provide a common isotropic resolution of $8 \mathrm{~mm}$ full width at half maximum resolution. These processed PET image sets are then uploaded back to LONI in a consistent Digital Imaging and Communications in Medicine (DICOM) format (Fig. 1). To date, the quality control failure rate is $11 \%$, which has declined from the initial year of the project when it was above $15 \%$, to less than $7 \%$ for the 18- and 24-month scans. The majority of scan failures was due to problems with image reconstruction and could be corrected with reprocessing; less than $2 \%$ of scans require rescanning.

\section{FDG data analysis in core PET laboratories}

Four Core laboratories have analyzed PET data. Three different approaches were taken to the FDG data. One involved use of prespecified regions of interest (ROIs), another used 3dimensional stereotactic surface projection (3D-SSP) with Neurostat, a free, noncommercial analysis program [2], and another lab took a voxelwise approach to the data using statistical parametric mapping (SPM) with separate training and validation data sets. The fourth laboratory performed analyses of the PIB data.

\subsection{ROI approaches (UC Berkeley)}

The methodological approach to the ROI-based data analysis involved the preselection of specific ROIs. This was done by identifying regions cited frequently in FDG-PET studies of $\mathrm{AD}$ and MCI patients. We conducted a metaanalysis in PubMed using all permutations of the following

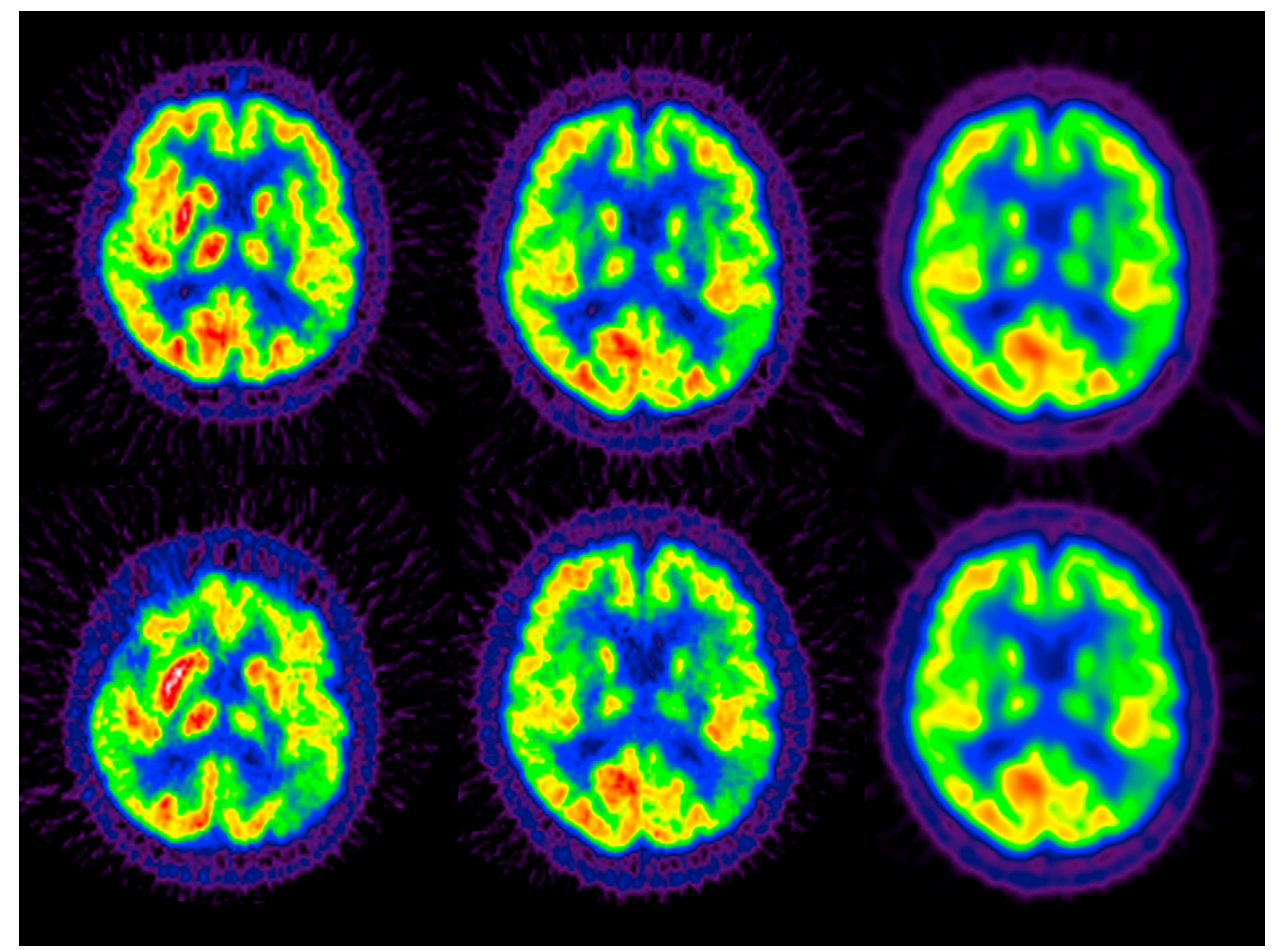

Fig. 1. Examples of the Alzheimer's Disease Neuroimaging Initiative (ADNI) processing stream. Top row: Baseline $\left[{ }^{18}\right.$ F]fluorodeoxyglucose positron emission tomography (FDG-PET) scan, mild cognitive impairment (MCI) subject. Bottom row: month 36 FDG scan, same subject. Left: FDG-PET image in native format

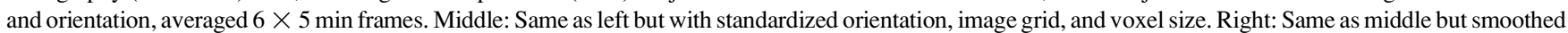
to an isotropic resolution of $8 \mathrm{~mm}$ full width at half maximum. Note longitudinal decrease seen in left parietal cortex (image right) in middle and right columns. 
search terms: AD or Alzheimer's; MCI or Mild Cognitive Impairment; FDG-PET or FDG or glucose metabolism. Within the studies identified by these terms, we isolated those that listed coordinates representing results of cross-sectional and/or longitudinal voxelwise analyses in which FDG uptake differed significantly between groups, changed in the same individuals over time, or correlated with cognitive performance. This resulted in a total of 292 Montreal Neurological Institute or Talairach coordinates and (if available) their accompanying $Z$-scores or $t$-values. The list of studies used to generate these FDG-ROIs is available online as supplementary data to our report on FDG-clinical correlations [3]. All coordinates were transformed into Montreal Neurological Institute template space. Then intensity values were generated for coordinates that reflected a combination of the $Z$-score or $t$-value associated with the coordinate and the degree to which coordinates within the same region overlapped (indicating repeated citations of the same region across studies). To do this, all $t$-values were transformed to approximate $Z$ scores. Then, overlapping $Z$ scores, when they occurred, were added. The volumes were smoothed with a $14 \mathrm{~mm}$ full width at half maximum smoothing kernel. Finally, the volume was intensity normalized using the maximum value, resulting in a map with values between 0 and 1 . The crosssectional coordinate map was then thresholded at 0.50 , and this resulted in a set of five regions located in right and left angular gyri, bilateral posterior cingulate gyrus, and left middle/inferior temporal gyrus (Fig. 2). Because these five ROIs were highly correlated, we generated a composite ROI by averaging across each subject. This composite ROI was used in the majority of subsequent analyses.

As noted, one of the original goals of ADNI was to investigate imaging as a biomarker of disease progression. Initial results were recently reported [3] and will be briefly summarized. In essence, we found that in a large sample (approximately 400 subjects comprised of normals, MCI, and AD patients with multiple time points up to 2 years) low glucose metabolism at baseline predicted decline on the Alzheimer's Disease Assessment Scale-Cognitive Subscale (ADAS-cog), and that longitudinal decline in glucose metabolism was associated with longitudinal decline on the ADAS-cog. We also determined that in the setting of a clinical trial, longitudinal metabolic measurements would have greater statistical

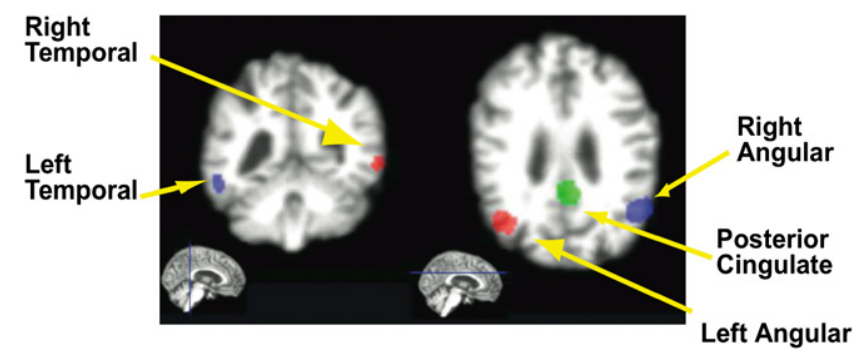

Fig. 2. These regions of interest (ROIs) were generated as described in the text using clusters of significant voxels from the FDG-PET literature. These were averaged together into a composite ROI for the ROI-based analyses. power for the detection of change than would longitudinal ADAS-cog measurements. For FDG-PET, the number of AD subjects per group to detect a $25 \%$ treatment change over 12 months was 180 subjects per arm (power $=0.80$, $\alpha=.05$, two tailed), which was substantially less than for the ADAS-cog.

The availability of multiple bioimarkers in ADNI participants provides a way of comparing these biomarkers to one another. In a recent publication [4], we investigated relationships between PIB-PET, FDG-PET, and cerebrospinal fluid (CSF) biomarkers ( $\mathrm{A} \beta_{42}$ and tau). A unique aspect of the study was the dichotomization of these biomarkers as "+" and "-" for AD using cutoffs obtained in samples that were distinct from ADNI, and then application of these cutoffs to the ADNI sample. In summary, we found high agreement between PIB and CSF A $\beta_{42}(91 \%$ agreement, $\kappa=.74)$, but generally modest to low agreement between PIB and other biomarkers. In contrast, FDG-PET was highly correlated with scores on the mini-mental state examination (MMSE) $(R=.63, P<.0001)$, whereas both PIB and CSF $\mathrm{A} \beta_{42}$ were not. Together these findings indicate that different biomarker measures of $A \beta$ are in agreement, but that non- $A \beta$ biomarkers are better indicators of disease status.

\subsection{Voxel-based approaches (Banner Alzheimer Institute)}

Voxel-based analysis of FDG-PET data used the automated brain mapping algorithm SPM5 (http://www.fil.ion. acl.ac.uk/spm/). Along the way, several new image-analysis methods were developed. SPM5 was used to generate statistical maps of between-group differences in regional-to-whole brain measurements of the cerebral metabolic rate for glucose (CMRgl) in probable $\mathrm{AD}, \mathrm{MCI}$, and normal control (NC) groups; correlations with categorical and continuous measurements of clinical disease severity; and differences between $A P O E \& 4$ carriers and noncarriers in each of these groups [5]. In comparison with NC subjects, AD and MCI patients each had significantly lower CMRgl bilaterally in posterior cingulate, precuneus, parietotemporal and frontal cortex, as well as occipital cortex, which were correlated with more severe dementia (measured with the Clinical Dementia Rating (CDR) and MMSE). In comparison with $A P O E \& 4$ noncarriers in the same subject group, $A P O E \& 4$ carriers in the NC group had significantly lower CMRgl in several $\mathrm{AD}$-affected regions, supporting previous findings from this group [6,7] and others [8,9]. Talairach brain atlas coordinates and measurements from the maximally significant locations were uploaded to the LONI website as a shared resource.

SPM5 was also used to characterize 12-month regionalto-whole brain $\mathrm{CMRgl}$ declines in the $\mathrm{AD}, \mathrm{MCI}$, and $\mathrm{NC}$ groups and provided the foundation to develop the statistical ROI (sROI) strategy and estimate the number of $\mathrm{AD}$ and $\mathrm{MCI}$ patients needed to evaluate AD-slowing treatments in multicenter randomized clinical trials (RCTs) as described later in the text. The AD and MCI groups each have significant 
12-month CMRgl declines bilaterally in posterior cingulate, medial and lateral parietal, medial and lateral temporal, and frontal and occipital cortex (Fig. 3), which were significantly greater than those in the $\mathrm{NC}$ group and correlated with measures of clinical decline.

Using a voxelwise approach, we developed an "empirically pre-defined statistical region-of-interest (sROI)" strategy, providing a single imaging endpoint with improved statistical power to evaluate $\mathrm{AD}$-slowing treatments in RCTs, freedom from multiple regional comparisons, and the ability to customize the endpoint to the subject group and between-scan interval of interest using voxel based image analysis techniques. sROI's were empirically predefined for each subject group and between-scan interval in a training data set from ADNI and then used in ADNI's independent testing data set to demonstrate its power to track sROI-toreference region $\mathrm{CMRgl}$ declines and evaluate $\mathrm{AD}$-slowing treatments with a fraction of the number of $\mathrm{AD}$ and $\mathrm{MCI}$ patients needed using clinical endpoints in 12-, 18- and 24-month parallel-group, placebo-controlled RCTs. These results indicated the ability to detect a $25 \%$ treatment effect in 12 months (power $=0.80, \alpha=0.05$, two tailed), with
$61 \mathrm{AD}$ patients per arm using FDG-PET as an outcome variable, as compared with 612 subjects using the ADASCog. For MCI, an identically powered study over 18 months would require 121 subjects per arm [10].

Also using a voxelwise approach, we developed a "hypometabolic convergence index (HCI)" strategy to automatically capture the AD-related pattern and magnitude of an individual's CMRgl reductions relative to a normal control group, providing a single measurement that might be used in the differential diagnosis, detection and tracking of $\mathrm{AD}$. The HCI was associated with categorical and continuous measurements of AD severity, better power than other biomarker measurements to predict 18-month progression from MCI to probable AD. Using data from another longitudinal study, the HCI was subsequently found to be associated with $A P O E \& 4$ gene dose in cognitively normal older adults.

Both the SROI and HCI strategies have the potential to be applied to other imaging modalities and voxel-based image analysis techniques. For instance, the sROI strategy was used to track the progression of $\mathrm{AD}$ with improved statistical power using structural magnetic resonance imaging (MRI) and tensor-based morphometry [11].

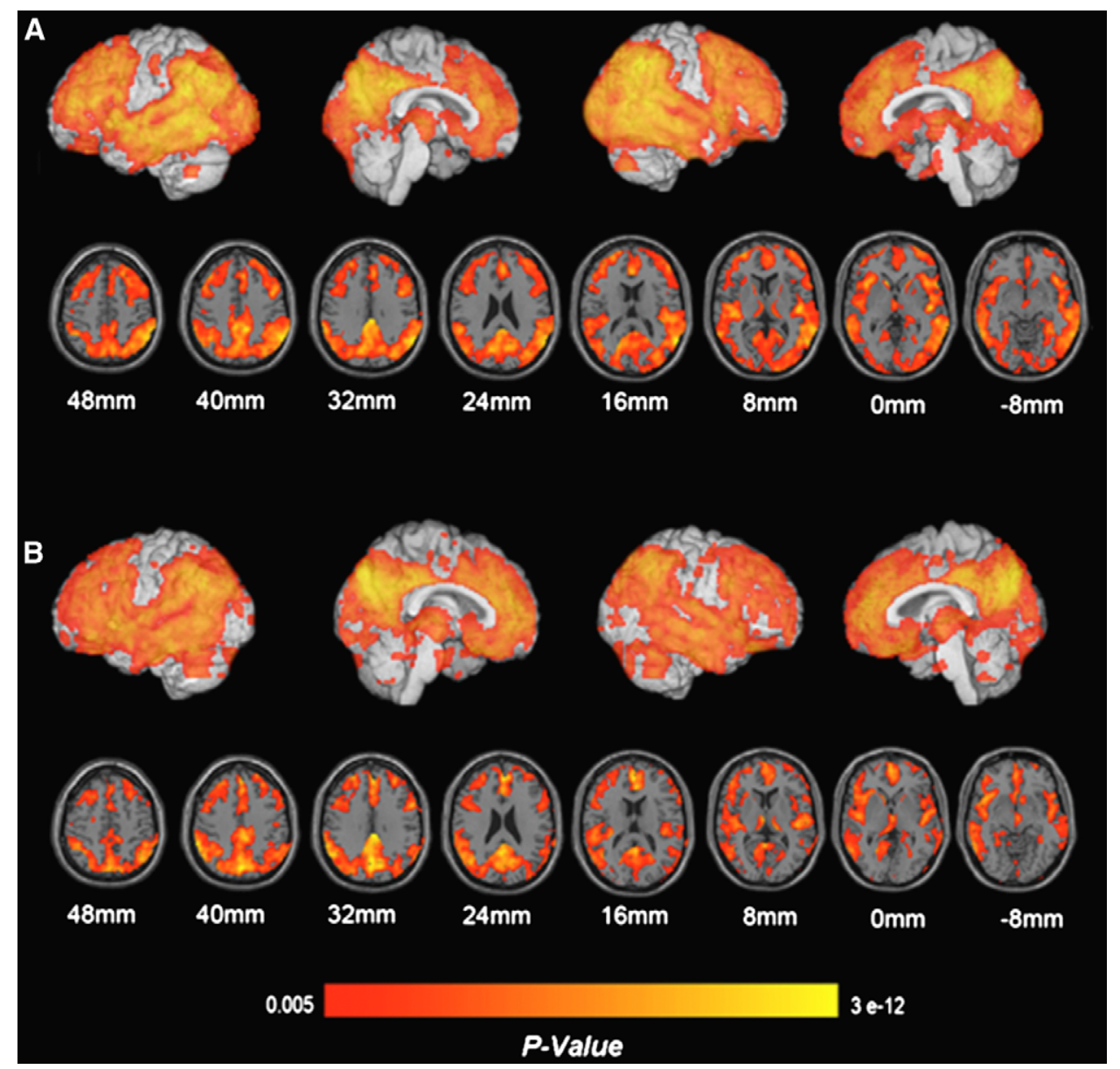

Fig. 3. Results of statistical parametric mapping analyses showing significant 12 month declines in glucose metabolism in patients with Alzheimer's disease (AD) (A) and MCI (B). 


\subsection{D-SSP approaches}

For these analyses, we utilized Neurostat to classify individual subjects and predict outcomes. Neurostat was designed specifically for the study of diseases of the cerebral cortex and to minimize the effects of focal brain atrophy on glucose metabolic rate. It realigns the PET image set to the anterior commissure-posterior commissure stereotactic coordinate system (Talaraich space) to ensure proper orientation for further processing. Next, Neurostat constructs thousands of vectors of fixed length perpendicular to the lateral and medial brain surface and searches for peak values to a depth of 6 voxels. Thus it generates approximately 16,000 predefined surface pixels covering the entire cortex, including the medial aspect of the two hemispheres. Neurostat also calculates the mean value from 20 predetermined anatomically defined regions in both hemispheres and in pons. By using peak values rather than rates derived from fixed coordinates, the mismatch of sulcal folds and partial volume effects of atrophy are minimized. These surface values are used to construct a comprehensive set of orthogonal SSP maps. Finally, metabolic images of glucose metabolism relative to the pons and images of $Z$-score values relative to metabolism seen in cognitively normal subjects are constructed for visual inspection and comparison with calculated regional values.

Numerical summary measures used to calculate sample sizes for a clinical trial, and to examine the relationship with cognitive measures were (1) the number of surface pixels with values $>2$ standard deviations (SD) below the normal control population, (2) the number of surface pixels with values $>3$ SD below the normal controls, (3) sum of $Z$ score values $>2$ SD below normal controls, (4) $Z$ score values based upon a regression of pons values in serial studies of an individual, (5) glucose metabolism relative to pons measured in a predetermined surface region representing the frontal cortex, and (6) glucose metabolism relative to pons averaged in frontal, parietal, and temporal association cortices. Numerical summary measures 1-4 are the PET Core's only measures of topographic extent of glucose metabolism, a novel outcome. These summary measures and Neurostat images warped into Talaraich space are available on the LONI website for interested investigators.

The Neurostat approach is especially suited for classification of individual images, which we have previously demonstrated can differentiate $\mathrm{AD}$ and frontotemporal dementia (FTD), showing specificity of the FTD pattern of hypometabolism using 3D-SSP of $>97 \%$ [12]. Using this approach in ADNI, we found that $10 / 93(10.8 \%)$ of AD subjects in ADNI clearly have an FTD pattern of hypometabolism. Similar analyses performed in ADNI normal and MCI subjects find occasional patients with patterns suggesting FTD or AD. We have suggested that misdiagnosis may be contaminating many $\mathrm{AD}$ research studies, and FDG-PET could improve the diagnostic accuracy of subjects enrolled in clinical trials [13]. Interestingly, the proportion of subjects with an AD diagnosis and FTD pattern is similar to the proportion of PIB-AD patients.

Neurostat permitted the examination of the relationship between the topographic extent of glucose hypometabolism (measured with 3D-SSP) and subsequent diagnosis. We found that the topographic extent of hypometabolism at baseline was greater in $\mathrm{MCI}$ subjects who converted to $\mathrm{AD}$ by 1 year, than in MCI subjects who continued to be classified on clinical grounds as MCI. We found that variation in the number of abnormal pixels largely reflected the timing of conversion, with patients who converted at 6 months having a greater extent of hypometabolism than those who converted later. Perhaps not surprisingly, converters had an AD-like

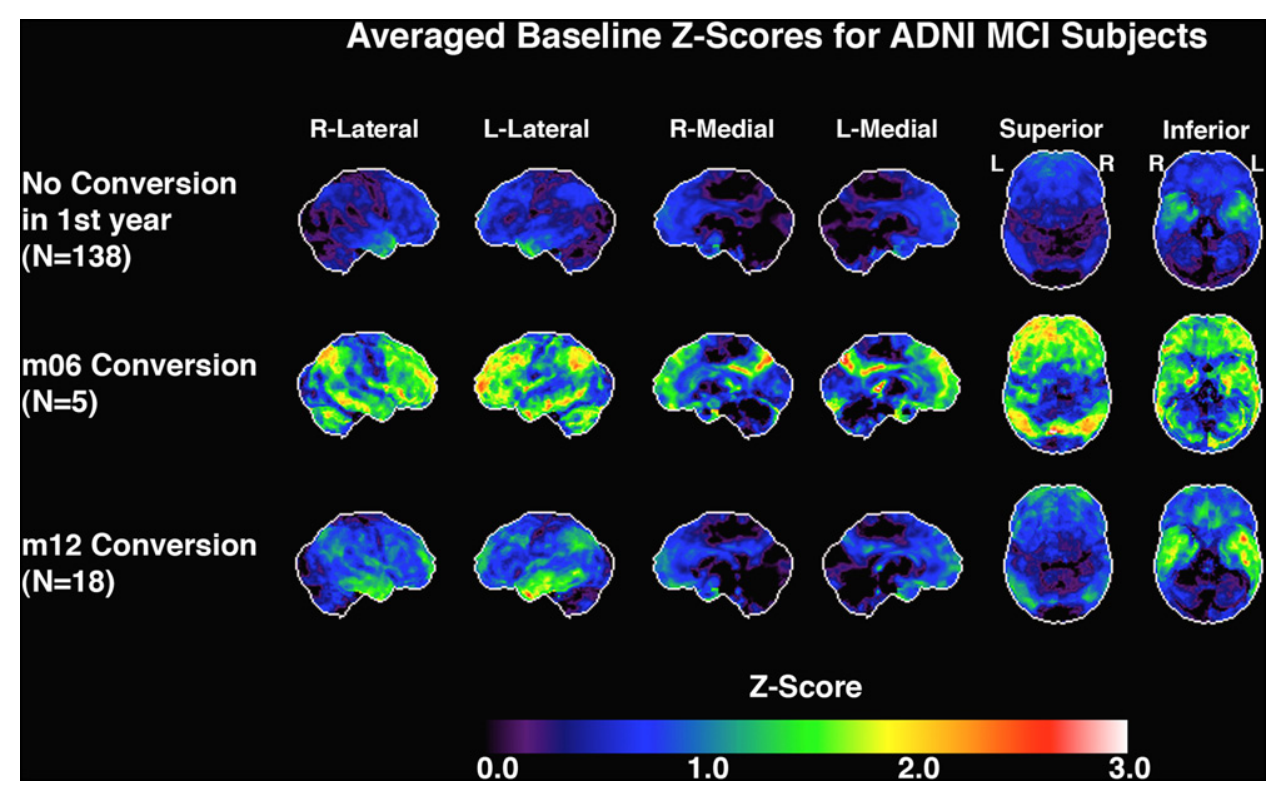

Fig. 4. SSP Images in ADNI MCI subjects by diagnosis at 12 months after imaging. 
pattern of hypometabolism. The pattern of average $Z$-scores in nonconverters shows little abnormality, whereas 6-month converters show more extensive hypometabolism than 12-month converters (Fig. 4).

\section{Analysis of amyloid imaging with $\left[{ }^{11} \mathrm{C}\right] \mathrm{PIB}$}

The PIB-PET add-on study began its third year in May 2009. To date, 103 baseline PIB-PET studies have been completed at 14 participating ADNI PET centers in 19 elderly cognitively normal controls (mean age $=78$, MMSE $=$ 29), 65 MCI subjects (mean age $=75$, MMSE $=27$ ), and 19 AD subjects (mean age $=73$, MMSE $=22$ ).

Regional assessment of the PIB-PET data involved sampling 13 different brain areas using an automated region of interest (ROI) template method. The template analysis method was based on the MRI of an elderly MCI subject (79 years), who was purposely chosen to represent a moderate degree of atrophy. Each subject's MRI scan was warped into MCI-Template space and then the same parameters were applied to the subject's PIB-PET scan, resulting in alignment of the MRI and PIB-PET to the MCI-Template. The MCITemplate ROIs were then used to sample the warped PIBPET images. Fig. 5 shows regional brain PIB-PET values in all 103 subjects in four cortical ROIs. In addition, a four region average (4 Reg Avg) value of all four ROIs was calculated for each subject. Positive amyloid deposition (PIB+) was defined when the SUVR (standardized uptake value ratio in the region relative to cerebellar gray matter) exceeded a regional value of 1.50 in the four region average (red line in Fig. 5). This PIB(+) cut-off value was based on studies in a large group of cognitively normal control subjects studied at the University of Pittsburgh and was described in a recent paper [14]. The PIB-PET data from these ADNI studies were generally consistent with literature reports from a variety of laboratories throughout the world. The control subjects

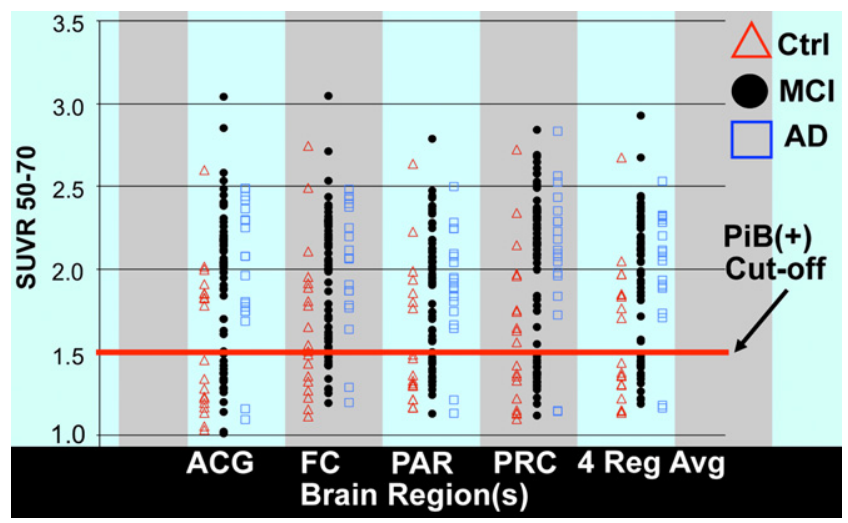

Fig. 5. Regional Pittsburgh compound B (PIB) PET SUVR outcome measure values in four brain regions (ACG, anterior cingulate; FC, frontal cortex; PAR, parietal cortex; and PRC, precuneus) and a four cortical regions averaged value (four Region Average) for all 103 PIB-PET baseline subjects in the ADNI PIB add-on studies. The cut-off value of 1.50 SUVR units dichotomizes all of the subjects into two groups: $\mathrm{PIB}(+)$ and $\mathrm{PIB}(-)$. Subjects are normal (red triangles), $\mathrm{AD}$ (blue boxes), and MCI (black circles). were comprised of two subgroups: one subgroup with relatively high SUVR values exceeding the $\mathrm{PIB}(+)$ cut-off value of 1.50 (9 of 19) and another subgroup with relatively low SUVR values below the cut-off value (10 of 19) (Fig. 5). Most literature studies using PIB have shown the frequency of amyloid-positivity in elderly cognitive control subjects (average age, 80 years) to be in the range of $30 \%$. In the ADNI PIB-PET cohort, we found a higher frequency of PIB-positivity of $47 \%$. Although the prevalence of PIB positivity is high, as shown above there is high congruence between PIB and CSF A $\beta$ measures [4]. As these control subjects will be studied longitudinally over the next few years, it will be interesting to determine whether their PIBpositivity becomes more robust and whether this change correlates with changes in cognition. The AD group showed PIB-positivity in 17 of 19 subjects (89\%). This seems to be typical of many centers throughout the world, which find that $10 \%-20 \%$ of their clinically diagnosed AD subjects are PIB-negative. Whether this is a reflection of inaccurate clinical diagnosis or the failure of PIB to detect amyloid deposits in a small fraction of $\mathrm{AD}$ subjects must await future post-mortem confirmation studies. Finally, the MCI group was comprised of an apparent continuum of PIB values, ranging from very PIB-negative to very PIB-positive with a number of subjects in an intermediate zone around the cut-off value of 1.50 SUVR units. For the ADNI MCI cohort, 18 of 65 MCI subjects were PIB(-) (28\%) and 47 of 65 MCI subjects were $\mathrm{PIB}(+)(72 \%)$. These values agree with the literature, which typically shows about $65 \%$ of MCI subjects studied to be $\mathrm{PIB}(+)$.

As of January 2010, 80 PIB-PET 1 year and 34 PIB-PET 2 year longitudinal ADNI studies have been completed. Data from all 1 year longitudinal studies have been processed by the University of Pittsburgh PET Group and posted on the LONI website (Fig. 6). These include 17 cognitively normal

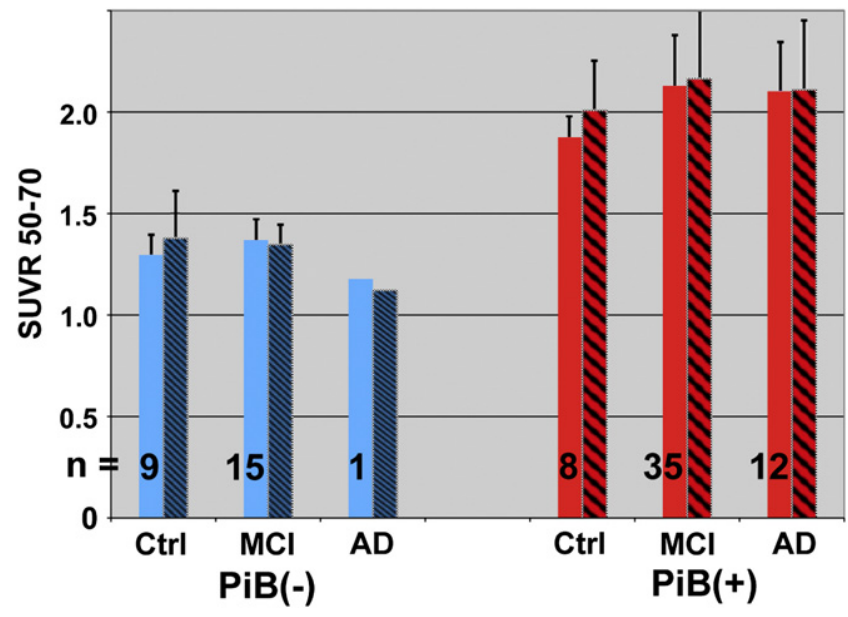

Fig. 6. Four region average SUVR values for 80 PIB-PET 1 year longitudinal follow-up subjects separated into control, $\mathrm{MCI}$, and $\mathrm{AD}$ groups, and each group further subdivided into $\mathrm{PIB}(-)$ and $\mathrm{PIB}(+)$ subgroups. $\mathrm{n}$ is the number of subjects in each subgroup and hatched boxes indicate the 1 year follow-up subgroup SUVR averages. 
Table 1

Number of $\mathrm{PIB}(+)$ and $\mathrm{PIB}(-)$ subjects showing significant increases in four region average SUVR values over 1 year of follow-up

\begin{tabular}{|c|c|c|c|c|c|c|}
\hline & \multicolumn{2}{|l|}{ Controls } & \multicolumn{2}{|l|}{ MCI } & \multicolumn{2}{|l|}{$\mathrm{AD}$} \\
\hline & Number & $\begin{array}{l}>0.215 \\
\text { SUVR }\end{array}$ & Number & $\begin{array}{l}>0.215 \\
\text { SUVR }\end{array}$ & Number & $\begin{array}{l}>0.215 \\
\text { SUVR }\end{array}$ \\
\hline $\operatorname{PIB}(+)$ & 8 & 3 & 35 & 4 & 12 & 3 \\
\hline $\operatorname{PIB}(-)$ & 9 & 2 & 15 & 0 & 1 & 0 \\
\hline
\end{tabular}

control, $50 \mathrm{MCI}$, and $13 \mathrm{AD}$ subjects. In all subject groups, there were small and insignificant changes in group average SUVR values over 1 year of follow-up. These results are consistent with literature reports of group average changes over 2 years $[15,16]$. However, if individual subjects are followed longitudinally, significant changes over time can be discerned. Using reliable change index methods [17] to track changes in individual subjects has been helpful in this regard. For application of the reliable change index method to the PIB-PET longitudinal cohort, measurement variability was determined as the standard deviation from test-retest PIB-PET data from the University of Pittsburgh, comprised of 22 subjects (seven controls, nine MCI, and six AD) who received two PIB-PET scans within 28 days. The four Region Average test-retest SUVR standard deviation of these data was 0.13 SUVR units. Hence, a change cut-off value of 0.215 SUVR units (delta-SUVR value) was determined to be a significant change in the four Region Average SUVR
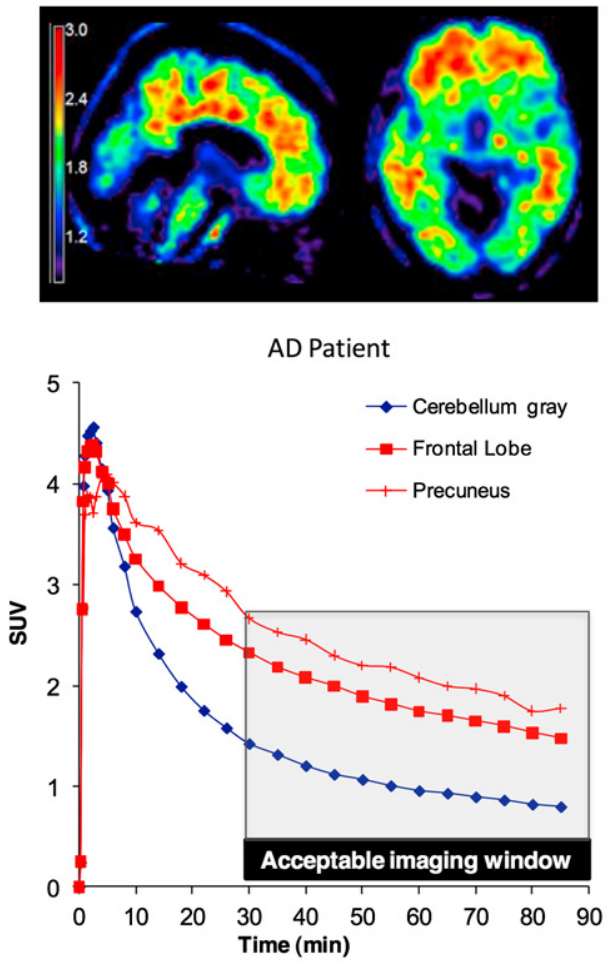

value of any subject with a confidence level of $95 \%$ and a $Z$-score of 1.645. The number of $\mathrm{PIB}(+)$ and $\mathrm{PIB}(-)$ subjects with significant increases in the four Region Average SUVR value ( $>0.215$ SUVR units) over 1 year of followup is shown in the Table 1. Across all subject groups, the number of significant $\mathrm{PIB}(+)$ subjects showing increases was 12 of 55 subjects $(22 \%)$, whereas only two of $25(8 \%)$ $\mathrm{PIB}(-)$ subjects increased. The group with the largest number of changes over 1 year of follow-up were the $\mathrm{PIB}(+)$ controls, in which three of eight subjects (38\%) showed significant increases.

\section{Future plans for ADNI}

The next activities of the PET core in ADNI will focus on developing and implementing similar protocols for amyloid imaging using the $\left[{ }^{18} \mathrm{~F}\right]$ labeled amyloid imaging agent AV45. Because of its longer half life $\left[{ }^{18 \mathrm{~F}}\right] \mathrm{AV} 45$ can be delivered to virtually all ADNI sites from existing commercial radiopharmacies. $\left[{ }^{18} \mathrm{~F}\right] \mathrm{AV} 45$ is currently in FDA Phase III studies for use as an amyloid imaging agent. Preclinical data on this compound have been reported [18], and it has many favorable properties including a dissociation constant of $3.7 \pm 0.3 \mathrm{nM}$, well within the range of other effective compounds ( $\left[{ }^{11} \mathrm{C}\right] \mathrm{PIB}$ has a $\mathrm{K}_{\mathrm{d}}$ of $\left.2 \mathrm{nM}\right)$.

Nineteen clinical trials have been completed or are currently underway with AV45, and more than 1000 subjects have undergone AV45-PET imaging worldwide. Studies so
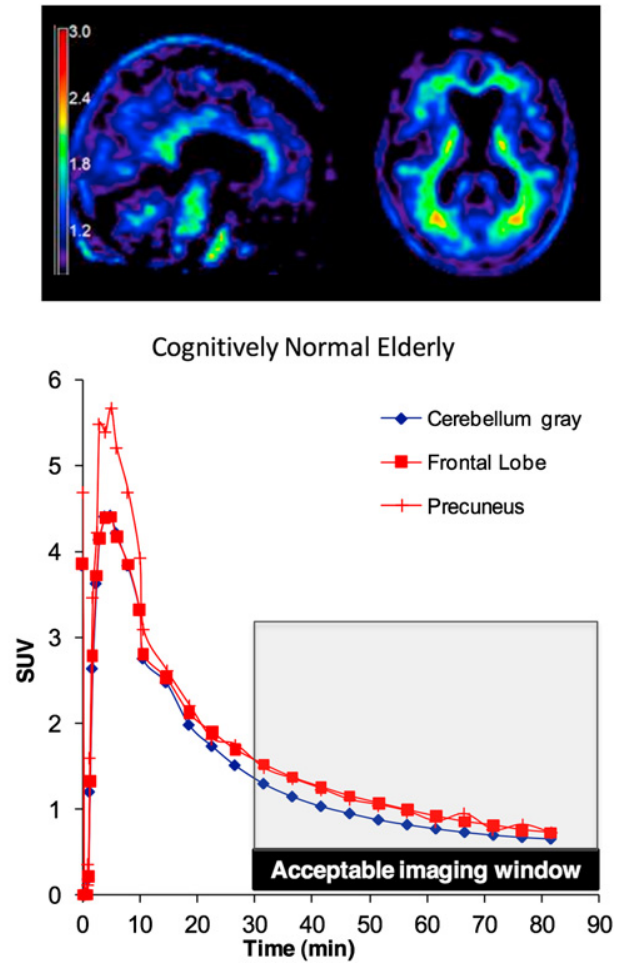

Fig. 7. Top: 10 min PET data from AV-45 in an AD patient (left) and healthy control (right) showing uptake in cortex in AD and nonspecific retention in white matter in the control. Bottom: Kinetics that demonstrate rapid uptake and washout with retention in cortical regions in the AD patient, washout in the same regions in controls, and similar washout in white matter and cerebellum in both subjects. 
far show favorable qualities such as safety, brain uptake, pharmacokinetics, and amyloid imaging properties. As shown in Fig. 7, the kinetics are favorable, reaching steady state relatively early so that images obtained relatively soon after injection can be used for quantitation. This factor combined with the longer half life of $\left[{ }^{18} \mathrm{~F}\right]$ makes short imaging times (10-20 $\mathrm{min})$ at about an hour after injection a very practical method of obtaining data for quantitation. Images obtained using equilibrium models, late images, or late standardized uptake value ratios (with cerebellum as a denominator) all show a pattern of binding identical to that seen with other amyloid tracers such as $\left[{ }^{11} \mathrm{C}\right] \mathrm{PIB}$ - that is, retention in cortical regions in $\mathrm{AD}$ patients that is most notable in precuneus/posterior cingulate, medial inferior frontal cortex, and other regions of association cortex (Fig. 7).

In the next phase of ADNI, all participants will receive amyloid imaging with $\left[{ }^{18} \mathrm{~F}\right] \mathrm{AV} 45$, and at the same time point these subjects will be studied with FDG-PET as well as MRI and CSF and blood biomarkers. By following these subjects over time, the relative changes in these biomarkers at different disease stages will be defined, paving the way for future clinical trials and interventions.

\section{Acknowledgments}

Data collection and sharing for this project was funded by the Alzheimer's Disease Neuroimaging Initiative (ADNI) (National Institutes of Health Grant U01 AG024904). ADNI is funded by the National Institute on Aging, the National Institute of Biomedical Imaging and Bioengineering, and through generous contributions from the following: Abbott, AstraZeneca AB, Bayer Schering Pharma AG, Bristol-Myers Squibb, Eisai Global Clinical Development, Elan Corporation, Genentech, GE Healthcare, GlaxoSmithKline, Innogenetics, Johnson and Johnson, Eli Lilly and Co., Medpace, Inc., Merck and Co., Inc., Novartis AG, Pfizer Inc, F. Hoffman-La Roche, Schering-Plough, Synarc, Inc., and Wyeth, as well as nonprofit partners the Alzheimer's Association and Alzheimer's Drug Discovery Foundation, with participation from the U.S. Food and Drug Administration. Private sector contributions to ADNI are facilitated by the Foundation for the National Institutes of Health (http://www.fnih.org/). The grantee organization is the Northern California Institute for Research and Education, and the study is coordinated by the Alzheimer's Disease Cooperative Study at the University of California, San Diego. ADNI data are disseminated by the Laboratory for Neuro Imaging at the University of California, Los Angeles. This research was also supported by NIH grants P30 AG010129, K01 AG030514, and the Dana Foundation.

\section{References}

[1] Joshi A, Koeppe RA, Fessler JA. Reducing between scanner differences in multi-center PET studies. Neuroimage 2009;46:154-9.
[2] Minoshima S, Frey KA, Koeppe RA, Foster NL, Kuhl DE. A diagnostic approach in Alzheimer's disease using three-dimensional stereotactic surface projections of fluorine-18-FDG PET. J Nucl Med 1995; 36:1238-48.

[3] Landau SM, Harvey D, Madison CM, Koeppe RA, Reiman EM, Foster $\mathrm{NL}$, et al. Associations between cognitive, functional, and FDG-PET measures of decline in $\mathrm{AD}$ and MCI. Neurobiol Aging (in press).

[4] Jagust WJ, Landau SM, Shaw LM, Trojanowski JQ, Koeppe RA, Reiman EM, et al. Relationships between biomarkers in aging and dementia. Neurology 2009;73:1193-9.

[5] Langbaum JB, Chen K, Lee W, Reschke C, Bandy D, Fleisher AS, et al. Categorical and correlational analyses of baseline fluorodeoxyglucose positron emission tomography images from the Alzheimer's Disease Neuroimaging Initiative (ADNI). Neuroimage 2009; 45:1107-16.

[6] Reiman EM, Caselli RJ, Yun LS, Chen K, Bandy D, Minoshima S, et al. Preclinical evidence of Alzheimer's disease in persons homozygous for the $\epsilon 4$ allele for apolipoprotein E. N Engl J Med 1996; 334:752-8.

[7] Reiman EM, Chen K, Alexander GE, Caselli RJ, Bandy D, Osborne D, et al. Functional brain abnormalities in young adults at genetic risk for late-onset Alzheimer's dementia. Proc Natl Acad Sci USA 2004; 101:284-9.

[8] Small GW, Mazziotta JC, Collins MT, Baxter LR, Phelps ME, Mandelkern MA, et al. Apolipoprotein E type 4 allele and cerebral glucose metabolism in relatives at risk for familial Alzheimer's disease. JAMA 1995;273:942-7.

[9] Small GW, Ercoli LM, Silverman DH, Huang SC, Komo S, Bookheimer SY, et al. Cerebral metabolic and cognitive decline in persons at genetic risk for Alzheimer's disease. Proc Natl Acad Sci USA 2000;97:6037-42.

[10] Chen K, Langbaum JBS, Fleisher AS, Reschke C, Liu X, Ayutyanont N, et al. Twelve-month metabolic declines in probable Alzheimer's disease and amnestic mild cognitive impairment using an empirically predefined statistical region-of-interest: findings from the Alzheimer's Disease Neuroimaging Initiative. Neuroimage (in press).

[11] Hua X, Lee S, Yanovsky I, Leow AD, Chou YY, Ho AJ, et al. Optimizing power to track brain degeneration in Alzheimer's disease and mild cognitive impairment with tensor-based morphometry: an ADNI study of 515 subjects. Neuroimage 2009;48:668-81.

[12] Foster NL, Heidebrink JL, Clark CM, Jagust WJ, Arnold SE, Barbas NR, et al. FDG-PET improves accuracy in distinguishing frontotemporal dementia and Alzheimer's disease. Brain 2007; 130:2616-35.

[13] Foster NL, Wang AY, Tasdizen T, Fletcher PT, Hoffman JM, Koeppe RA. Realizing the potential of positron emission tomography with $18 \mathrm{~F}$-fluorodeoxyglucose to improve the treatment of Alzheimer's disease. Alzheimers Dement 2008;4:S29-36.

[14] Aizenstein HJ, Nebes RD, Saxton JA, Price JC, Mathis CA, Tsopelas ND, et al. Frequent amyloid deposition without significant cognitive impairment among the elderly. Arch Neurol 2008; 65:1509-17.

[15] Engler H, Forsberg A, Almkvist O, Blomquist G, Larsson E, Savitcheva I, et al. Two-year follow-up of amyloid deposition in patients with Alzheimer's disease. Brain 2006;129:2856-66.

[16] Mathis CA, Price JC, Klunk WE, Weissfield L, McNamee R, Wolk DA, et al. Longitudinal PIB measures in control, MCI, and AD subjects. J Nucl Med 2008;49(Suppl 1):35P.

[17] Jacobson NS, Truax P. Clinical significance: a statistical approach to defining meaningful change in psychotherapy research. J Consult Clin Psychol 1991;59:12-9.

[18] Choi SR, Golding G, Zhuang Z, Zhang W, Lim N, Hefti F, et al. Preclinical properties of ${ }^{18} \mathrm{~F}-\mathrm{AV}-45$ : a PET imaging agent for A-beta plaques in the brain. J Nucl Med 2009;50:1887-94. 